\title{
DETERMINAÇÃO DE COMPOSTOS FENÓLICOS POR CROMATOGRAFIA LÍQUIDA DE ALTA EFICIÊNCIA ISOCRÁTICA DURANTE ESTACIONAMENTO DA ERVA-MATE
}

\author{
Fabiana L. Goularte Dutra e Rosemary Hoffmann-Ribani* \\ Departamento de Engenharia Química, Universidade Federal do Paraná, CP 19011, 81531-970 Curitiba - PR, Brasil \\ Marcelo Ribani \\ Instituto de Tecnologia do Paraná, Rua Prof. Algacir M. Mader, 3775, 81350-010 Curitiba - PR, Brasil
}

Recebido em 14/2/09; aceito em 9/6/09; publicado na web em 13/11/09

\begin{abstract}
DETERMINATION OF PHENOLIC COMPOUNDS BY ISOCRATIC HIGH PERFORMANCE LIQUID CHROMATOGRAFIC METHOD DURING STORAGE OF YERBA-MATE. Different phenolic compound, 5- caffeoylquinic acid (5-CQA), caffeic acid $(\mathrm{AC})$ and rutin $(\mathrm{Ru})$ contents of yerba-mate (Ilex paraguariensis) Brazilian samples of 06 different regions of São Mateus - Paraná, during natural and accelerated industrial storage, were evaluated. For quantification, a reverse phase HPLC isocratic method was developed and validated using methanol:water $(35: 65 \mathrm{v} / \mathrm{v})$ acidified with $0.5 \%$ acetic acid as mobile phase and a photodiode array detector. The six sample global average contents were (34.90 and $\left.36.10 \mathrm{mg} \mathrm{g}^{-1}\right)$ for 5-CQA, $\left(0.18 \mathrm{mg} \mathrm{g}^{-1}\right.$ and $\left.0.23 \mathrm{mg} \mathrm{g}^{-1}\right)$ for AC and (7.12 and $7.18 \mathrm{mg} \mathrm{g}^{-1}$ ) for $\mathrm{Ru}$, respectively, for the natural and accelerated storage systems. The results showed that the 5-CQA and $\mathrm{Ru}$ content are kept constant during the storage while AC content increase only during accelerated storage.
\end{abstract}

Keywords: HPLC analysis; phenolic compounds; Ilex paraguariensis.

\section{INTRODUÇÃO}

A erva-mate (Ilex paraguariensis Saint Hilaire) é uma espécie nativa brasileira, destacando-se como fonte econômica, social e ecológica para a região sul do Brasil, norte e leste da Argentina e Paraguai, sendo consumida principalmente na forma de chimarrão. ${ }^{1,2}$

Vários efeitos benéficos à saúde humana têm sido atribuídos ao consumo da erva-mate. Em estudos in vitro, o extrato de erva-mate contribuiu na prevenção do câncer. ${ }^{3,4}$ Estudos in vivo com infusão de erva-mate demonstraram a capacidade de inibição da auto-oxidação do LDL (lipoproteína de baixa densidade), ${ }^{5}$ a eficácia no tratamento contra obesidade ${ }^{6}$ e ação cardioprotetora.

Os benefícios atribuídos ao consumo da infusão de erva-mate estão relacionados aos compostos fenólicos, metabólitos secundários produzidos pelas plantas, ${ }^{8}$ que atuam como antioxidantes, ${ }^{9}$ sendo oxidados em preferência a outros constituintes do alimento ou componentes celulares e tecidos. ${ }^{10}$ Estão presentes na erva-mate, dentre outros compostos, a rutina $(\mathrm{Ru})$, um flavonol pertencente à classe dos flavonoides e os ácidos 5-cafeoilquínico (5-CQA) e cafeico (AC), pertencente aos ácidos fenólicos. ${ }^{11,12}$

Filip et al. ${ }^{13}$ correlacionaram a atividade antioxidante da infusão de erva-mate com conteúdo de rutina, quercetina, kampferol e derivados cafeoilquínicos desta.

De acordo com a literatura, ${ }^{14-18}$ os teores em base seca de Ru, AC e 5-CQA encontrados na erva-mate, variam de 0,60 a 13,00 $\mathrm{mg} \mathrm{g}^{-1}$; 0,14 a $0,37 \mathrm{mg} \mathrm{g}^{-1}$ e 5,70 a 28,00 $\mathrm{mg} \mathrm{g}^{-1}$, respectivamente. O conteúdo destes compostos na erva-mate é fator importante para estimular o apelo ao consumo do produto, sendo que os trabalhos mostram que existem variações nos teores, para um mesmo componente, em função da localidade e modo de cultivo, ${ }^{15}$ ou processamento empregado na industrialização. ${ }^{19}$

Além desses fatores, também se observam diferenças nos métodos para a extração dos compostos fenólicos em erva-mate, podendo ser por infusão aquosa, ${ }^{15}$ hidrólise ácida ${ }^{20}$ ou maceração da amostra ${ }^{18}$ seguida de ebulição sob refluxo ${ }^{16,21}$ com solução hidroalcoólica.

*e-mail: ribani@ufpr.br
A cromatografia líquida de alta eficiência (CLAE) tem sido a técnica mais empregada na análise de compostos fenólicos em ervamate, utilizando principalmente gradiente da fase móvel para separação de diferentes classes de compostos, como os flavonoides e ácidos hidroxicinâmicos, principais componentes presentes na erva-mate..$^{714,22}$

Muitos laboratórios de instituições disponibilizam sistemas CLAE equipados apenas com bomba simples, de menor custo, e empregando eluição isocrática, mantendo constante o fluxo da fase móvel na coluna, por ser um sistema de fácil operação e com boa reprodução entre diferentes marcas de equipamentos.

O processamento industrial da erva-mate envolve diferentes etapas que podem modificar sua composição química e alterar o sabor do produto final. O armazenamento ou estacionamento é uma etapa da industrialização que visa levar à formação de componentes aromáticos e à transformação da $\operatorname{cor}^{23}$ verde da erva-mate para amarela, não apreciada pelo consumidor brasileiro, mas uma exigência do principal mercado importador do Brasil, o Uruguai. ${ }^{24}$ Esse pode ser realizado de forma natural onde a erva-mate cancheada é armazenada em depósitos por períodos que variam entre 6 meses a 2 anos, sob condições ambientais, ou acelerada na qual a erva-mate é mantida em câmaras com temperatura e umidade relativa controladas, por um período de 30 a 60 dias. $^{25}$

Além da composição química rica em metilxantinas e saponinas, os compostos cafeoilquínicos compõem até $12 \%$ do peso seco na erva-mate, ${ }^{26}$ indicando sua importância na possível determinação do sabor do produto final. Adicionalmente, a percepção de adstringência da bebida de erva-mate tem sido correlacionada positivamente com o conteúdo de polifenois totais, ${ }^{27}$ sendo que para os produtos brasileiros, atualmente, não existem dados publicados para os compostos fenólicos em erva-mate durante estacionamento/armazenagem.

O objetivo deste trabalho foi verificar a influência dos sistemas industriais de estacionamento natural e acelerado em 6 diferentes amostras de erva-mate brasileira para exportação, no teor de compostos fenólicos. Para análise destes foi desenvolvido e validado um método cromatográfico isocrático de separação das diferentes classes de compostos fenólicos como a Ru, um flavonol e os 5-CQA e AC, derivados hidroxicinâmicos, presentes na erva-mate. 


\section{PARTE EXPERIMENTAL}

\section{Amostras}

A erva-mate utilizada no estudo foi fornecida pela Ervateira Baldo S.A. Comércio, Indústria e Exportação, da Unidade de São Mateus do Sul, Paraná. Seis amostras de erva-mate cancheadas, de diferentes produtores da região, foram selecionadas aleatoriamente, divididas em dois lotes, acondicionadas em sacos de $\pm 45 \mathrm{~kg}$ de polipropileno (gramatura $0,062 \mathrm{~g} \mathrm{~m}^{-2}$ ) e, posteriormente, submetidas aos processos de estacionamento, natural e acelerado.

No estacionamento acelerado (EA), amostras de erva-mate ficaram armazenadas em câmaras, sob condições controladas, com umidade relativa variando entre 31,4 e $34,5 \%$ e temperatura de 48,9 a $51,4{ }^{\circ} \mathrm{C}$ por um período de 60 dias, durante o qual foram coletados $5 \mathrm{~kg}$ de cada amostra no tempo inicial (zero), aos 10, 20, 30, 40, 50 e 60 dias para análise.

As amostras de erva-mate cancheadas submetidas ao estacionamento natural (EN) foram acondicionadas em galpão construído em alvenaria, na cidade de São Mateus do Sul, sob condições de temperatura variando de 24,15 a $26,00^{\circ} \mathrm{C}$ e umidade relativa ambiente variando de 31,40 a 57,69\%. Este armazenamento foi realizado por um período de 6 meses, de setembro de 2007 a fevereiro de 2008, sendo retirados $5 \mathrm{~kg}$ de cada amostra no tempo inicial (zero) e aos 30, 60, 90, 120, 150 e 180 dias para análise.

\section{Umidade}

Cada amostra coletada foi analisada quanto à umidade conforme a metodologia descrita pelo IAL em Brasil, ${ }^{28}$ que consiste na secagem direta em estufa a $105^{\circ} \mathrm{C}$.

\section{Reagentes}

Os padrões analíticos de Ru, AC e 5-CQA foram adquiridos da Sigma Chemicals Co. (St. Louis, EUA). O metanol grau cromatográfico e o ácido acético de grau analítico foram adquiridos da Merk (Darmstadt, Alemanha). Utilizou-se um sistema Milli-Q da Millipore (Bedford, USA) para purificar a água utilizada para o preparo das soluções padrão e fase móvel. Foi utilizada membrana filtrante com poros de $0,45 \mu \mathrm{m}$, da Millipore.

\section{Extração dos compostos fenólicos}

A extração dos compostos da erva-mate foi realizada de acordo com Ribani, ${ }^{21}$ adaptada. Consistiu em adicionar $100 \mathrm{~mL}$ de solução água:etanol 1:1 (v/v) em $2 \mathrm{~g}$ de erva-mate mantida por $12 \mathrm{~h}$, a temperatura ambiente. Seguiram-se três extrações com $25 \mathrm{~mL}$ da solução hidro-etanólica a 50\% sob refluxo por 30 min cada. Recolheram-se todos os extratos em balão volumétrico de $250 \mathrm{~mL}$ completando-se o volume. Cada hidrolisado foi filtrado em membrana filtrante, antes da análise cromatográfica.

\section{Preparo dos padrões}

Soluções estoque dos padrões foram preparadas pela diluição de cada composto fenólico em metanol com concentrações de aproximadamente 1000, 2000 e $1000 \mu \mathrm{g} \mathrm{mL}^{-1}$, para AC , 5-CQA e Ru, respectivamente.

\section{Estabelecimento das condições cromatográficas para CLAE}

O trabalho foi conduzido em cromatógrafo a líquido Merck Hitachi, equipado com injetor automático L-7250. O sistema de detecção utilizado foi UV-VIS com varredura espectral L-7455 de 200 a $500 \mathrm{~nm}$, monitorado em 325 para detecção do AC e 5-CQA e $370 \mathrm{~nm}$ para a Ru. A análise foi conduzida utilizando-se uma coluna Bondclone ${ }^{\circledR} \mathrm{C}-18,10 \mu \mathrm{m}(3,9 \mathrm{x}$ $300 \mathrm{~mm}$ ) da Phenomenex, com volume de injeção de $10 \mu \mathrm{L}$, utilizando metanol e água, acidificado com $0,5 \%$ de ácido acético, como fase móvel. A temperatura da coluna foi mantida a $30^{\circ} \mathrm{C}$ durante o período da análise e a vazão da fase móvel foi mantida constante a $1 \mathrm{~mL} \mathrm{~min}^{-1}$. O Software Merck HSM, versão 4.1 foi utilizado para o tratamento dos dados.

Para definir a condição cromatográfica, visando a separação dos compostos fenólicos, inicialmente utilizou-se uma solução contendo a mistura dos padrões Ru, AC e 5-CQA composta por alíquotas tomadas das soluções estoque dos padrões. Testaram-se diferentes proporções de fase móvel aquosa com metanol contendo $0,5 \%$ de ácido acético, até se estabelecer a melhor separação entre os padrões, que foi utilizada como base para definir a separação para a erva-mate, com e sem adição de padrões. A quantificação nos extratos das amostras foi realizada por padronização externa e as determinações de cada amostra foram conduzidas em duplicata.

\section{Validação do método}

A seletividade foi avaliada pela comparação dos tempos de retenção dos picos obtidos na separação frente aos dos padrões e dos espectros no início, meio e fim de cada pico, com o dos respectivos padrões em um banco de dados, utilizando-se isto como uma indicação da presença do composto puro.

A linearidade do método por cromatografia foi verificada para $\mathrm{Ru}$, AC e 5-CQA na faixa de 0,4 a 5,0; 10,6 a 170,1 e 20,9 a 628,7 $\mu \mathrm{g} \mathrm{mL} \mathrm{m}^{-1}$, respectivamente. As curvas analíticas foram construídas pela injeção em triplicata das soluções padrão de trabalho em cinco concentrações diferentes, baseadas nas faixas esperadas dos seus teores na erva-mate.

Os limites de detecção (LD) e quantificação (LQ) foram definidos pelos parâmetros das curvas de calibração, sendo calculados como a mínima concentração correspondente a 3,3 x (SD/S) e 10 x (SD/S) respectivamente, sendo SD o erro do coeficiente linear e $\mathrm{S}$ a inclinação da curva analítica. ${ }^{29}$

Para verificar a precisão do método, valores de seis repetições da mesma amostra foram analisados quanto a sua repetitividade, expressada através do coeficiente de variação $(\mathrm{CV})$. $^{30}$

A avaliação da exatidão do método foi segundo o processo de fortificação com padrões, sendo o número de ensaios estabelecido conforme recomendação da ANVISA, ${ }^{30}$ verificada para três níveis de concentração dos padrões, em triplicata. Prepararam-se nove repetições de 2,0 g da mesma amostra de erva-mate, conforme descrito em Extração dos compostos fenólicos, adicionadas dos padrões antes da extração. Foi adicionado em cada nível 250,0; 500,0 e 1000,0 $\mu \mathrm{L}$ da solução padrão de 1886,0 $\mu \mathrm{g} / \mathrm{mL}$ do 5-CQA. A partir da solução padrão do AC com 20,5 $\mu \mathrm{g} / \mathrm{mL}$, foi adicionada a cada nível 105,0; 210,0 e 1000,0 $\mu \mathrm{L}$. Para a Ru foi adicionado 200,0; 400,0 e 1000,0 $\mu \mathrm{L}$ da solução estoque de 1063,0 $\mu \mathrm{g} / \mathrm{mL}$. Assim as quantidades de cada padrão adicionadas em cada nível foram, respectivamente, de 472,0; 943,0 e 1886,0 $\mu$ g para 5-CQA; 2,2;

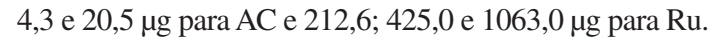

\section{Análise estatística}

Os dados foram submetidos à análise de variância e as médias comparadas pelo teste de Tukey. O programa utilizado para análise estatística foi o Statistix versão 8.0. ${ }^{31}$

\section{RESULTADOS E DISCUSSÃO}

\section{Validação do método cromatográfico}

A separação do extrato de erva-mate, com e sem adição de padrões, foi conduzida sob a melhor condição obtida para a solução 
contendo a mistura dos padrões. A proporção da fase móvel aplicada variou de 15:85 (metanol:água) até 42:58 (metanol:água), sempre com 0,5\% de ácido acético. Observou-se que na proporção de metanol inferior a $30 \%$ a Ru ficou retida na coluna. Para a proporção de até $34 \%$ de metanol a separação do ácido cafeico e 5-CQA foi eficiente, entretanto, nessas condições, na análise da amostra, o pico da $\mathrm{Ru}$ apresentou-se sobreposto a um outro componente da erva-mate. A separação do AC, 5-CQA e Ru na amostra foi otimizada utilizandose fase móvel metanol e água (35:65, v/v) acidificados com $0,5 \%$ de ácido acético.

Por meio da comparação dos tempos de retenção e dos espectros dos picos obtidos na separação com os correspondentes tempos de retenção e espectros dos padrões, confirmou-se a separação eficiente do flavonol Ru, e dos derivados hidroxicinâmicos, 5-CQA e AC, dos demais componentes presentes na erva-mate estudada (Figura 1), comprovando a seletividade da separação.
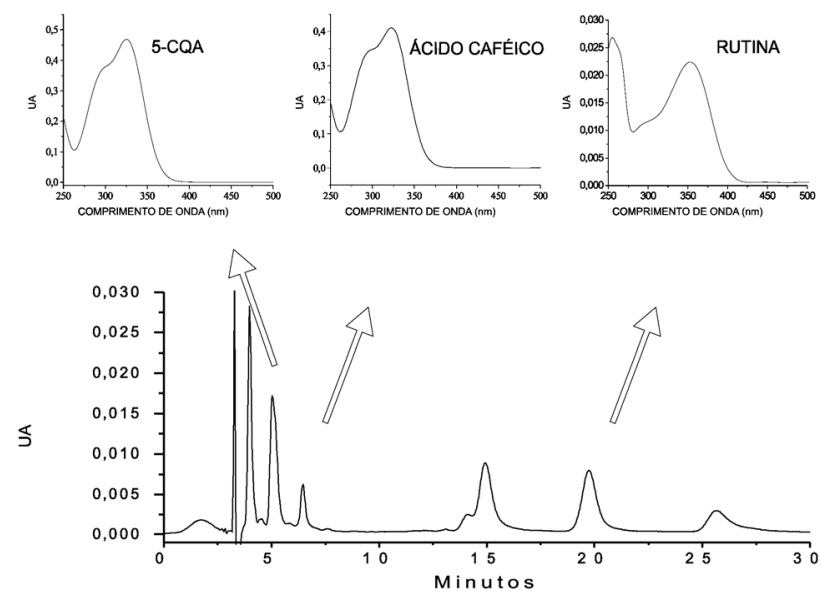

Figura 1. Cromatograma dos padrões dos compostos fenólicos adicionados à amostra de erva-mate e respectivos espectros (250 a $500 \mathrm{~nm}$ ) obtidos do detector de arranjo de diodos. Condições da análise cromatográfica: coluna Bondclone ${ }^{\circledR}$ C-18 (3,9 $\left.\times 300 \mathrm{~mm}, 10 \mu \mathrm{m}\right)$ da Phenomenex, utilizando como fase móvel metanol:água (35:65, v/v), acidificado com 0,5\% de ácido acético e vazão constante de 1,0 $\mathrm{mL} \mathrm{min}^{-1}$, detecção a $370 \mathrm{~nm}$

As curvas analíticas apresentaram-se lineares na faixa de 0,41 a 5,00; 10,63 a 170,08 e 20,96 a 628,74 $\mu \mathrm{g} \mathrm{mL}^{-1}$, respectivamente, para o AC, Ru e 5-CQA com coeficientes de determinação respectivos de 0,9998; 0,9984 e 0,9980. Os limites de detecção do método obtidos pelos parâmetros das curvas analíticas foram: 11,12; 288,78 e 295,68 $\mathrm{mg} \mathrm{kg}^{-1}$, respectivamente, para AC, Ru e 5-CQA.

A precisão (repetitividade) obtida resultou em CV de 2,96; 1,79 e 2,07\%, respectivamente, para Ru, AC e 5-CQA. A exatidão do método foi demonstrada pela média dos valores obtidos na recuperação em percentagem de 95,6; 97,3 e 95,7\%, respectivamente, para $\mathrm{Ru}$, $A C$ e 5-CQA.

\section{Compostos fenólicos em erva-mate estacionada nos sistemas, natural e acelerado}

Todos os resultados dos conteúdos dos compostos analisados são apresentados em base seca, pois durante o estacionamento ocorreu diferença significativa nos teores da umidade das amostras que variaram de 7,78 a $11,12 \%$ e 7,07 a $10,45 \%$ para os estacionamentos natural e acelerado, respectivamente.

As seis amostras, quando analisadas por localidade de cultivo, apresentaram variações significativas para as médias nos teores dos compostos fenólicos ao longo dos estacionamentos, porém sem uma tendência definida para a variação. Como cada amostra foi proveniente de misturas de distintas progênies e partes da árvore, sob incidência solar diferente, considerou-se que estes fatores, conforme estabelecido por Edreva, ${ }^{32}$ para os metabólicos secundários de plantas, podem ter influenciado na variação dos resultados.

Quanto ao tipo de estacionamento, de acordo com os resultados da análise de variância para Ru, 5-CQA e AC obtidos para a média geral das seis amostras, houve influência significativa apenas na variável ácido cafeico, Tabela 1 , cujo teor foi maior no estacionamento acelerado $\left(0,23 \mathrm{mg} \mathrm{g}^{-1}\right)$, quando comparado ao estacionamento natural $\left(0,18 \mathrm{mg} \mathrm{g}^{-1}\right)$.

Tabela 1. Médias dos teores de Ru, AC e 5-CQA, em amostras de erva-mate ${ }^{2}$, durante os estacionamentos natural e acelerado, calculados em base seca

\begin{tabular}{lccc}
\hline Tratamentos & $\mathrm{AC}\left(\mathrm{mg} \mathrm{g}^{-1}\right)$ & 5-CQA $\left(\mathrm{mg} \mathrm{g}^{-1}\right)$ & $\mathrm{Ru}\left(\mathrm{mg} \mathrm{g}^{-1}\right)$ \\
\hline $\begin{array}{l}\text { Estacionamento } \\
\text { natural }\end{array}$ & $0,18 \pm 0,05 \mathrm{~b}$ & $34,88 \pm 6,39 \mathrm{a}$ & $7,12 \pm 2,78 \mathrm{a}$ \\
$\begin{array}{l}\text { Estacionamento } \\
\text { acelerado }\end{array}$ & $0,23 \pm 0,07 \mathrm{a}$ & $36,08 \pm 4,78 \mathrm{a}$ & $7,18 \pm 2,27 \mathrm{a}$ \\
\hline
\end{tabular}

Médias seguidas pela mesma letra não diferem estatisticamente pelo teste de Tukey no nível de 5\% de probabilidade. ${ }^{2}$ Média de seis localidades \pm desvio padrão, totalizando 42 amostras.

De acordo com a Figura 2 observa-se que o conteúdo de AC durante o EA aumenta significativamente, confirmado pela análise de variância e demonstrado pelo teste de Tukey para as médias (Tabela 2).

Por hidrólise o AC pode ser liberado dos outros derivados cafeoilquínicos (3 e 4-cafeoilquínico e 3,4; 3,5 e 4,5-dicafeoilquínicos)
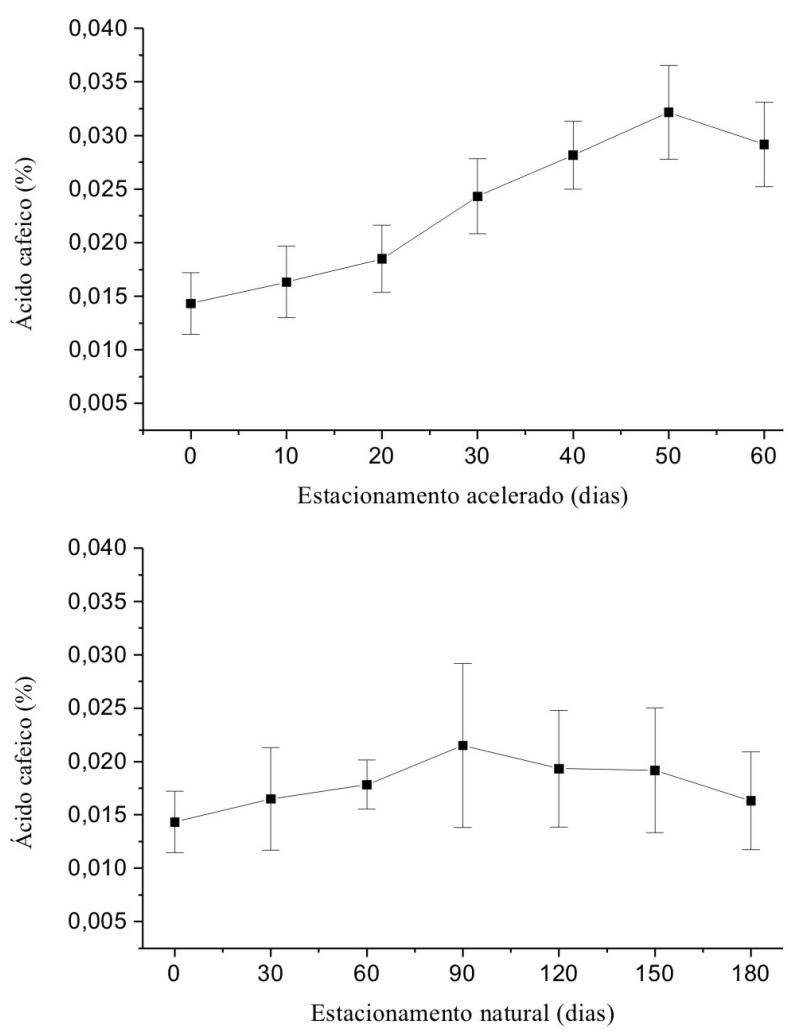

Figura 2. Conteúdo de ácido cafeico em amostras de erva-mate armazenada no estacionamento acelerado e natural em função do tempo 
Tabela 2. Médias dos teores de AC, em erva-mate durante estacionamento acelerado

Amostras $^{1}$

Estacionamento acelerado

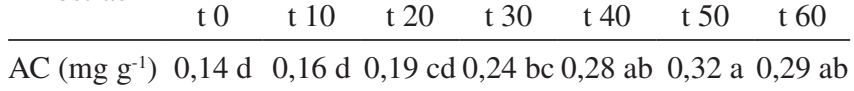

Médias seguidas por letras iguais não diferem significativamente pelo teste de Tukey a 5\% de probabilidade; $\mathrm{t}=$ Tempo (dias); ${ }^{1}$ Média de seis repetições

também presentes na erva-mate ${ }^{16}$ e que não foram monitorados neste experimento. $\mathrm{O}$ estudo de López et al ${ }^{17}$ demonstrou redução no teor do ácido 3,5-dicafeoilquínico para amostra de erva-mate analisada antes e após um EA.

Os valores obtidos de $\mathrm{AC}$ e Ru no presente trabalho variaram de 0,09 a $0,38 \mathrm{mg} \mathrm{g}^{-1}$ e, 3,20 a 12,70 $\mathrm{mg} \mathrm{g}^{-1}$, respectivamente, estando dentro da faixa de variação dos dados relatados pela literatura, Tabela 3.

Tabela 3. Teores de 5-CQA, AC e Ru expressos em base seca ( $\left.\mathrm{mg} \mathrm{g}^{-1}\right)$ em amostras de erva-mate analisadas por CLAE

\begin{tabular}{lcccc}
\hline Ref. & $\begin{array}{c}\text { Número de } \\
\text { Amostras }\end{array}$ & 5-CQA & AC & Ru \\
\hline $\begin{array}{l}\text { Presente } \\
\text { trabalho }\end{array}$ & $\mathrm{n}=6$ & 18,50 a 48,11 & 0,09 a 0,38 & 3,19 a 12,62 \\
14 & $\mathrm{n}=3$ & $28,0 \pm 3,0$ & $0,23 \pm 0,04$ & $0,60 \pm 0,05$ \\
15 & $\mathrm{n}=4$ & 7,3 a 12,7 & 0,34 a 0,37 & - \\
16 & $\mathrm{n}=5$ & 5,70 a 20,20 & - & \\
17 & $\mathrm{n}=1$ & $20,3 \pm 0,5$ & $0,35 \pm 0,02$ & $13,0 \pm 0,3$ \\
18 & $\mathrm{n}=16$ & 7,86 a 9,32 & 0,14 a 0,20 & - \\
$211^{\mathrm{a}}$ & $\mathrm{n}=6$ & 13 a 24,3 & - & 2,50 a 7,50 \\
33 & $\mathrm{n}=3$ & $17,99 \pm 0,77$ & $0,15 \pm 0,007$ & - \\
$34^{\text {a }}$ & $\mathrm{n}=7$ & 2,93 a 19,18 & 0,42 a 0,55 & - \\
\hline
\end{tabular}

${ }^{\mathrm{a}}$ Resultados apresentados em base úmida

Os valores obtidos para 5-CQA variaram nas amostras de 8,60 a $48,50 \mathrm{mg} \mathrm{g}^{-1}$, sendo a média geral $34,90( \pm 6,4)$ e 36,10 $( \pm 4,8) \mathrm{mg} \mathrm{g}^{-1}$, respectivamente, para erva-mate estacionada nos sistemas natural e acelerado e superior aos valores reportados na literatura para erva-mate (Tabela 3 ) e também superiores ao conteúdo para café, que segundo estudos apresenta teores de 1,14 a $17,85 \mathrm{mg} \mathrm{g}^{-1,35,36}$

O comportamento observado para $\mathrm{Ru}$ e 5-CQA durante EA, foi semelhante ao observado por López et al. ${ }^{17}$ que, comparando os teores em uma amostra de erva-mate Argentina, antes e após o armazenamento num sistema de EA, não constataram diferenças significativas nas concentrações destes compostos. Estudos com 5-CQA demonstram que este é estável quando submetido a $40^{\circ} \mathrm{C}$ por até $60 \mathrm{~min}$, com diminuição da estabilidade em função do aumento do tempo de exposição quando em temperatura superior a $100{ }^{\circ} \mathrm{C} . .^{37,38}$

\section{CONCLUSÕES}

As condições cromatográficas estabelecidas para separação no modo isocrático dos compostos fenólicos de diferentes classes (flavonoides de ácidos hidroxicinâmicos) em erva-mate foram: fase móvel metanol:água, na proporção de 35:65 (v/v), acidificado com 0,5\% ácido

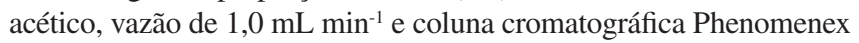
Bondclone ${ }^{\circledR}$ C-18 (3,9 x $\left.300 \mathrm{~mm}\right)$ com partículas de 10 micras.
Os teores obtidos para a média global das seis amostras foram concordantes aos dados relatados em outros trabalhos sendo 0,18 e 0,23 $\mathrm{mg} \mathrm{g}^{-1}$ para o AC e 7,12 e 7,18 $\mathrm{mg} \mathrm{g}^{-1}$ para Ru, respectivamente, para os sistemas de estacionamento natural e acelerado. As médias obtidas para o 5-CQA foram 34,90 e 36,10 $\mathrm{mg} \mathrm{g}^{-1}$, respectivamente, para EN e EA e superiores aos valores médios apresentados por outros autores.

O estudo demonstrou que os conteúdos da Ru e 5-CQA, presentes na erva-mate, não sofreram influência dos sistemas de estacionamento, enquanto que o teor do $\mathrm{AC}$ aumentou apenas no EA.

\section{AGRADECIMENTOS}

À CAPES pela concessão de bolsa à primeira autora, aluna do PPGTA; à Ervateira Baldo S.A. Comércio, Indústria e Exportação, Unidade de São Mateus do Sul, Paraná/Brasil pelo fornecimento das amostras e ao TECPAR pelo suporte técnico.

\section{REFERÊNCIAS}

1. Penteado, S. C. R.; Iede, E. T.; Leite, M. S. P.; Anais do $2^{\circ}$ Congresso sul-americano da erva-mate: $3^{a}$ Reunião técnica da erva-mate, Encantado, Brasil, 2000.

2. http://sistemasdeproducao.cnptia.embrapa.br/FontesHTML/Erva-mate/ CultivodaErvaMate/01_importancia_socioec.htm, acessada em Janeiro 2009.

3. Ramirez-Mares, M. V.; Chandra, S., Gonzalez de Mejia, E.; Mutat. Res. 2004, 554, 53.

4. Chandra, S.; Gonzales de Mejia, E.; J. Agric. Food Chem. 2004, 52, 3583.

5. Gugliucci, A.; Biochem. Biophys. Res. Commun. 1996, 224, 338.

6. Dickel, M. L.; Rates, S. M. K.; Ritter, M. R. J.; Ethnopharmacol. 2007, 109, 60 .

7. Menini, T.; Heck, C.; Schulze, J.; Mejia, E.; Gugliucci, A.; Planta Med. 2007, 73, 1141

8. Taiz, L.; Zeiger, E.; Fisiologia Vegetal, $3^{\text {a }}$ ed., Artmed: Porto Alegre, 2004.

9. Bravo, L.; Goya, L.; Lecumberri, E.; Food Res. Int. 2007, 40, 393.

10. Pelúzio, M. C. G.; Oliveira, V. P. Em Vitaminas Antioxidantes in Alimentos Funcionais; Costa, N. M. B.; Rosa, C. O. B., eds.; UFV: Viçosa, 2006.

11. Robards, K.; Antolivic, M.; Analyst 1997, 122, 11R.

12. Clifford, M. N.; Wu, W.; Kirkpatrick, J.; Kuhnert, N.; J. Agri. Food Chem. 2007, 55, 929

13. Filip, R.; Lotito, S. B.; Ferraro, G.; Fraga, C. G.; Nutr. Res. 2000, 20, 1437.

14. Filip, R.; Lopez, P.; Giberti, G.; Coussio, J.; Ferraro, G.; Fitoterapia 2001, 72, 774.

15. Streit, N. M.; Hecktheuer, L. H. R.; Canto, M. W.; Mallmann, C. A.; Streck, L.; Parodi, T. V.; Canterle, L. P.; Food Chem. 2007, 102, 560.

16. Clifford, M. N.; Food Chem. 1990, 35, 13.

17. López, P.; Isolabella, S.; Anesini, C.; Ferraro, G.; Filip, R.; Anais do $4^{\circ}$ Congreso Sudamericano de la Yerba Mate, Posadas, Argentina, 2006

18. Cardoso Júnior, E. L.; Ferrarese-Filho, O.; Cardoso Filho, L.; Ferrare, M. L. L.; Donaduzzi, C. M.; Sturion, J. A.; J. Food Composit. Anal. 2007, 20, 553.

19. Zanoelo, E. F.; Cardoso-Filho, L.; Cardoso-Júnior, E. L.; J. Food Process Eng. 2006, 29, 253.

20. Bastos, D. M.; Fornari, A. C.; Queiroz, Y. S.; Torres, E. A. F. S.; Braz. Arch. Biol. Technol. 2006, 49, 399.

21. Ribani, R. H.; Tese de Doutorado, Universidade Estadual de Campinas, Brasil, 2006.

22. Hoffmann-Ribani, R.; Rodriguez-Amaya, D. B.; Anais do $4^{\circ}$ Congreso Sudamericano de la Yerba Mate, Posadas, Argentina, 2006. 
23. Nietsche, K.; Dissertação de Mestrado, Universidade Federal do Paraná, Brasil, 2002.

24. http://www.seab.pr.gov.br/arquivos/File/PDF/agronegocio_erva_mate_ abr_2007.pdf, acessada em Novembro 2009.

25. De Bernardi, L. A., Prat Kricun, S. D.; Cadena alimentaria de "yerba mate" "Ilex paraguariensis" - Diagnóstico de la regíon yerbatera. Secretaria de Agricultura, Ganadería, Pesca y Alimentación: Buenos Aires, 2001

26. Schneider, E.; Scherer, R.; Urfer, P.; Janssens, M. J. J.; Anais do $4^{\circ}$ Congreso Sudamericano de la Yerba Mate, Posadas, Argentina, 2006.

27. Tamasi, O.; Filip, R.; Ferraro, G.; Calviño, A.; Anais do $4^{\circ}$ Congreso Sudamericano de la Yerba Mate, Posadas, Argentina, 2006.

28. Brasil, Ministério da Saúde, ANVISA; Métodos Físico-químicos para análise de alimentos - Normas analíticas do IAL, $4^{\mathrm{a}}$ ed., Ministério da Saúde: Brasília, 2005.

29. Ribani, M.; Bottoli, C. B. G.; Collins, C. H.; Jardim, I. C. S. F.; Quim. Nova 2004, 27, 771 .
30. Brasil, Ministério da Saúde, ANVISA; Guia para validação de métodos analíticos e bioanalíticos - Resolução RE n॰899 de 29/05/2003, Ministério da Saúde: Brasília, 2003.

31. Statistix ${ }^{\oplus}$; Statistixfor Windows: Analytical Software, Tallahas-see, EUA, 2003.

32. Edreva, A.; Agric. Ecosyst. Environ. 2005, 106, 135.

33. Marques, V.; Farah, A.; Food Chem. 2009, 113, 1370.

34. Bortoluzzi, A. L. M.; Pasqualato, R. P. R.; Guesser, G.; Cardoso Júnior, E. L.; Donaduzzi, C. M.; Mitsui, M.; Anais do $4^{\circ}$ Congreso Sudamericano de la Yerba Mate, Posadas, Argentina, 2006.

35. Nogueira, M.; Trugo, L. C.; Ciênc. Tecnol. Aliment. 2003, 23, 296.

36. Monteiro, M. C.; Trugo, L. C.; Quim. Nova 2005, 28, 637.

37. De Maria, C. A. B.; Trugo, L. C.; De Mariz e Miranda, L. S.; Salvador, E.; Food Res. Int. 1998, 31, 475.

38. Benincá, C.; Dissertação de Mestrado, Universidade Federal do Paraná, Brasil, 2009. 